\title{
Development of the loT-based Monitoring System for Scaffold Shoring System of Concrete Formwork
}

\author{
Jian $\mathrm{Su}^{1, \mathrm{a}}$, Jieming $\mathrm{Li}^{1}$, Lingsheng Tan ${ }^{1}$, Xiaodan Huang ${ }^{1}$ \\ ${ }^{1}$ Guangzhou Institute of Building Science Co., Ltd., Guangzhou, China
}

\begin{abstract}
Construction safety monitoring is an important approach to prevent collapse of scaffold shoring system during concrete formwork. In this article, the causes of the collapse of scaffold shoring system are investigated. A monitoring system based on the Internet of Things(IoT) is then proposed, consisting of wireless sensors, wireless monitor, wireless alarm, monitoring cloud platform and mobile client. The monitoring system is then put into practical application and has successfully detected the malfunction of the monitored system, which proves the efficiency and reliability of the monitoring system.
\end{abstract}

\section{Introduction}

Scaffold shoring systems are frequently used as temporary support during concrete placement. In recent years, with the increasing number of high-rise buildings and large span structures, the scaffold shoring systems in the constructions are also tend to be higher, larger, and bearing more load, which has significantly raised the risk of collapse. According to the statistics of the Ministry of Housing and Urban-Rural Development of the People's Republic of China, the percentage of collapse of scaffold shoring system has counted $29.63 \%$ in all types of construction accidents in 2016, causing the death of 30 workers. Therefore, it is important to conduct monitoring for the scaffold shoring system to ensure safety during concrete placement.

In most monitoring cases, only two parameters, lateral and vertical displacements of the scaffold, are monitored. And the monitoring equipment is basically optical instrument[1-2], such as level and total station, while the data needs to be collected manually. However, the collapse of scaffold shoring system usually happens in just a few seconds, manually data collection (once every $30 \mathrm{~min}$ ) cannot meet the requirement of forecasting the abrupt failure of the system. Moreover, since the optical path will be blocked by the tubes and slabs, only the displacements on the edge of the scaffold shoring system can be observed, which makes it difficult to prevent failure at the middle of the system.

Automatic optical instruments have been applied in scaffold monitoring to overcome the problems of manual data collection[3-4], but the blind area in the middle of the scaffold still exists. The method of installing sensors onto the scaffold for monitoring data collection is a good way to solve the problem, and have been put into practice in some tests and experiments for research purposes[5-6].

Based on the analysis of the scaffold shoring systems' failure mechanism, this paper proposes a monitoring method for scaffold shoring system during concrete formwork with the IoT technology, and then the method is applied to practical formwork project to verify its validity.

\section{Causes of the Scaffold Shoring System Collapses}

The collapses of the scaffold shoring system can be caused by the instability of the scaffold (buckling of the tubes) and the failure of the materials (breaking of the slabs, beam members, tubes or couplers). Normally, the critical buckling strength of the tube is lower than the yield strength of the steel, and the breaking of a certain component may eventually lead to the overall instability, so the instability of the scaffold shoring system is considered to be the main cause of the collapse. And the instability is affected by three factors: material, designing and assembling.

\subsection{Material factor}

When designing the scaffold shoring system, the parameters of each component of the system, such as material strength and size, are determined by designing code. However, in practice, it is very often that some of the materials are reused, so the actual value of these parameters may not reach the standard. Once the load exceeds the limit of the unqualified component, especially tubes, may cause the instability of the system.

In China, the $\phi 48 \mathrm{~mm} \times 3.5 \mathrm{~mm}$ tubes $(48 \mathrm{~mm}$ in diameter and $3.5 \mathrm{~mm}$ in wall thickness) are commonly used to build scaffold shoring systems, but according to the statistics carried out in the construction fields [8], the wall thickness of the tubes follows a model of normal distribution, with just an average of $3.29 \mathrm{~mm}$, and the minimum wall thickness counted is less than $2.5 \mathrm{~mm}$. The

*Corresponding author: ${ }^{a}$ Corresponding author: 691451947@qq.com 
bearing capacity for tubes suggested by Chinese designing code is

$$
N_{\max }=\frac{\pi \varphi f}{4}\left[D^{2}-(D-2 h)^{2}\right]
$$

Where $\varphi$ is a stability coefficient, $f$ is the compressive strength of steel, $D$ and $h$ stands for the diameter and wall thickness of the tube, respectively. Form Eq.(1), when the wall thickness of the tube is reduced from $3.5 \mathrm{~mm}$ to $2.5 \mathrm{~mm}$, the tube's bearing capacity will drop by $26.97 \%$.

Moreover, the reused tubes might have defects like bending and deformation as well, which will also lower the bearing capacity of the tubes and turn out to cause collapse.

\subsection{Designing factor}

Most scaffold shoring system are designed by the construction companies instead of professional designing companies. If the construction company did not pay enough attention or did not have enough knowledge about the scaffold shoring system, and just finish the designing based on the former pattern, may lead to incorrect designing, especially when the scaffold shoring system is high or large. The spacing between columns and the height of each storey are considered to be the key parameters in the designing of the system.

The spacing between columns determines the number of columns of the system. The larger the spacing is designed, the fewer columns are there to support the load, and thus more loads will be put onto each column, which makes the system less safe. With the bearing capacity of a single tube, the maximum surface load for the system can be derived as

$$
q_{\max }=\frac{\pi \varphi f}{4 l_{a} l_{b}}\left[D^{2}-(D-2 h)^{2}\right]
$$

Where $l_{a}$ and $l_{b}$ are the latitudinal and longitudinal spacing of the columns.

The height of each storey determines the effective length of the column, according to the critical load for instability of the tubes

$$
\sigma_{\mathrm{cr}}=\frac{\pi^{2} E I}{(\mu l)^{2}}
$$

Where $E$ and $I$ are the elastic modulus and the moment of inertia of the tube. And $\mu l$ is the effective length of the column. Eq.(3) shows that if the storey gets higher, the column is more likely to be instable.

\subsection{Assembling factor}

The assembly quality of the scaffold shoring system depends very much on the construction workers. With the absence of supervision, the scaffold is likely to be of poor assembly quality due to the varying skill levels of workers.
Over height of the top storey, suspending of columns, missing of the X-bracing, and low tightening torque of the coupler are the commonly observed assembly problems in the construction field. Numerical analysis[9] has proved the negative effect of these problems on the stability of the scaffold shoring system.

\section{THE IOT-BASED MONITORING SYSTEM FOR SCAFFOLD SHORING SYSTEM}

Based on the causes of the collapses of scaffold shoring system, four parameters should be monitored during concrete placement: slab settlement, overall lateral displacement, column axial force and column tilting angle. Among these parameters, the slab settlement is the most important, because both tube buckling or material breaking will eventually be reflected by slab settlement. Overall lateral displacement reflects the lateral deformation of the scaffold. The purpose of column axial force monitoring is to prevent the buckling of tubes due to overloading. And column tilting angle is the reflection of tubes' deformation and vibration under construction loads. In the monitoring system, all these parameters are measured by sensors and transmitted with IoT technology.

With the characteristic of the IoT technology, the monitoring system is designed to consist of wireless sensors, wireless monitor, wireless alarm, intelligent monitoring cloud platform and the wireless monitoring mobile client. The mechanism of the monitoring system is shown as Figure1. The wireless sensors collect the data of monitoring parameters from the scaffold shoring system, then the data is sent to the wireless monitor through ZigBee. The data is analysed and stored by the wireless monitor, and the result is displayed to the inspectors at the construction site in no time. The realtime monitoring result is sent to the intelligent monitoring cloud platform and the mobile client through $4 \mathrm{G}$ network, keeping the manager of the project be informed.

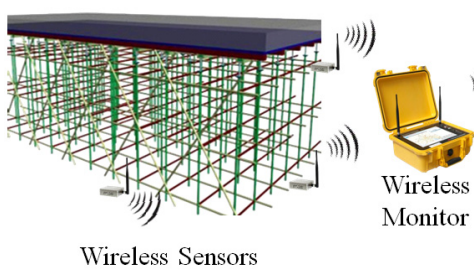

i)) Mobile Client

Figure 1. Cloud Platform

\subsection{Wireless sensors}

Different from the optical monitoring instruments, which can only monitor the rim of the scaffold from the outside, the wireless sensors can be installed in the scaffold, eliminating the blind area of optical monitoring. In this case, the entire scaffold shoring system can be monitored, making the monitoring more effective. Considering that it takes a lot of climbing on the scaffold to install the sensors, the sensors are designed to be wireless, thus the 
installation will be completely free of cables or tools. According to the monitoring parameters, three types of wireless sensors are developed:

(1) Wireless displacement sensor.

The wireless displacement sensor (Figure 2(a)) is used to collect the data of slab settlement and the scaffold horizontal displacement. A string is settled between the monitoring point and the benchmark. The displacement of the monitoring point changes the length of the string and is detected by the displacement sensor.

(2) Wireless pressure sensor

The wireless pressure sensor is used to monitoring the axial force imposed onto the column. Since the axial forces tend to get re-distributed between columns with the help of lateral tubes, it would be meaningless to monitor the axial forces at the bottom of the column. Thus, the wireless pressure sensor must be settled between the slab (sometimes beam member) and the Uhead of the column, as shown in Figure 2(b).

(3) Wireless angle sensor

The wireless angle sensor is used to detect the tilting or vibration of the column caused by the concentrated or dynamic load. With the top storey considered to be the weakest spot of the column, the angel sensor should be installed at the upper end of the tube with a fastener, as shown in Figure 2(c).

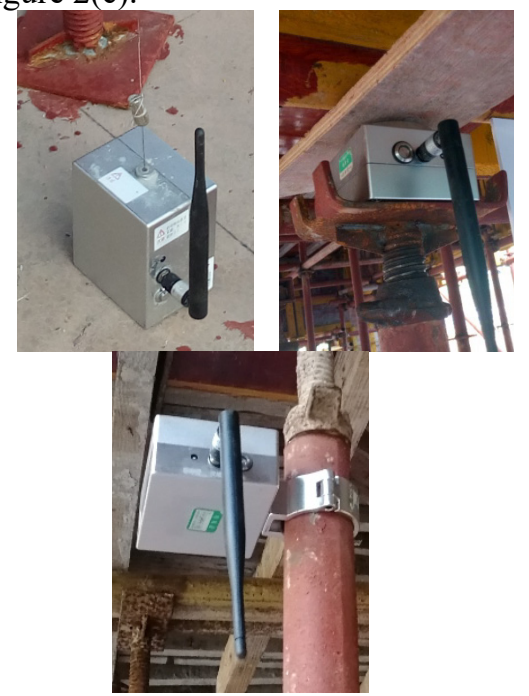

(a)displacemnt sensor(b)pressure sensor (c)angle sensor Figure 2. Wireless sensors

\subsection{Wireless monitor}

The wireless monitor (Figure 3) is designed with Android OS and is used to conduct real-time monitoring at the construction site. It has the functions of modifying and querying the project's information, laying out the monitoring points, checking the real-time and history monitoring data. Once deployed, the monitor is automatically connected with the wireless sensors and the alarm through ZigBee technology. When the collected monitoring data exceeds the alert value, the monitor triggers the alarm automatically.
The wireless alarm (Figure 4) is settled in the construction field to send out alarming sound and light to warn the workers when the monitoring data exceeds, so that they know that the scaffold shoring system is potentially unstable and evacuate immediately.

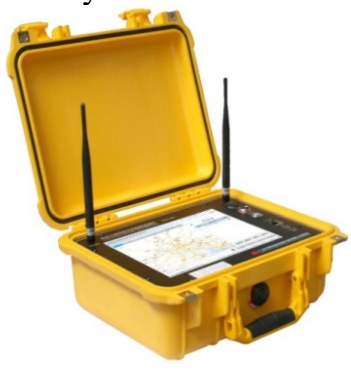

Figure 3. Wireless monitor

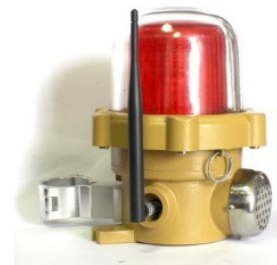

Figure 4. Wireless alarm

\subsection{Intelligent monitoring cloud platform}

The intelligent monitoring cloud platform is designed for remote monitoring and management. Developed with the $\mathrm{B} / \mathrm{S}$ structure and J2EE+SQLServer model, the cloud platform can realize remote project information maintenance, user management, real-time monitoring and report checking. The interface of the cloud platform is shown in Figure 5.

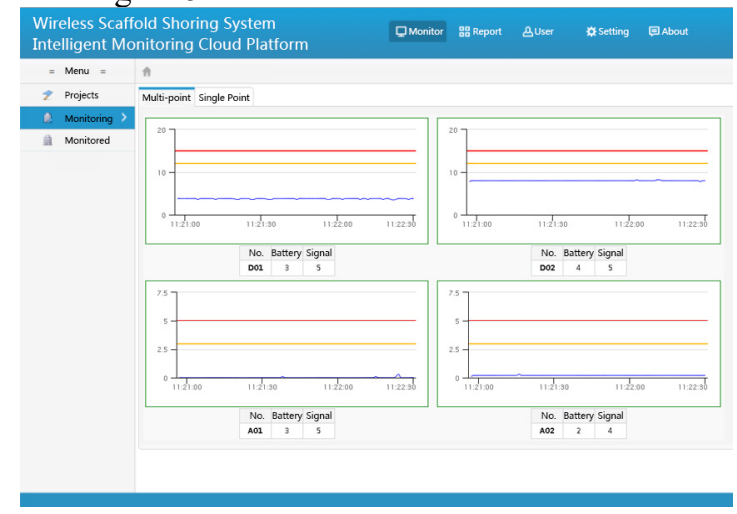

Figure 5. Intelligent monitoring cloud platform

\section{5 the wireless monitoring mobile client}

The intelligent monitoring cloud platform is designed for the managers to ensure they can be informed with the monitoring result at anytime and anywhere. The managers can receive the real-time monitoring data and the alarming message with the mobile client. The interface of the mobile client is shown in Figure 6. 


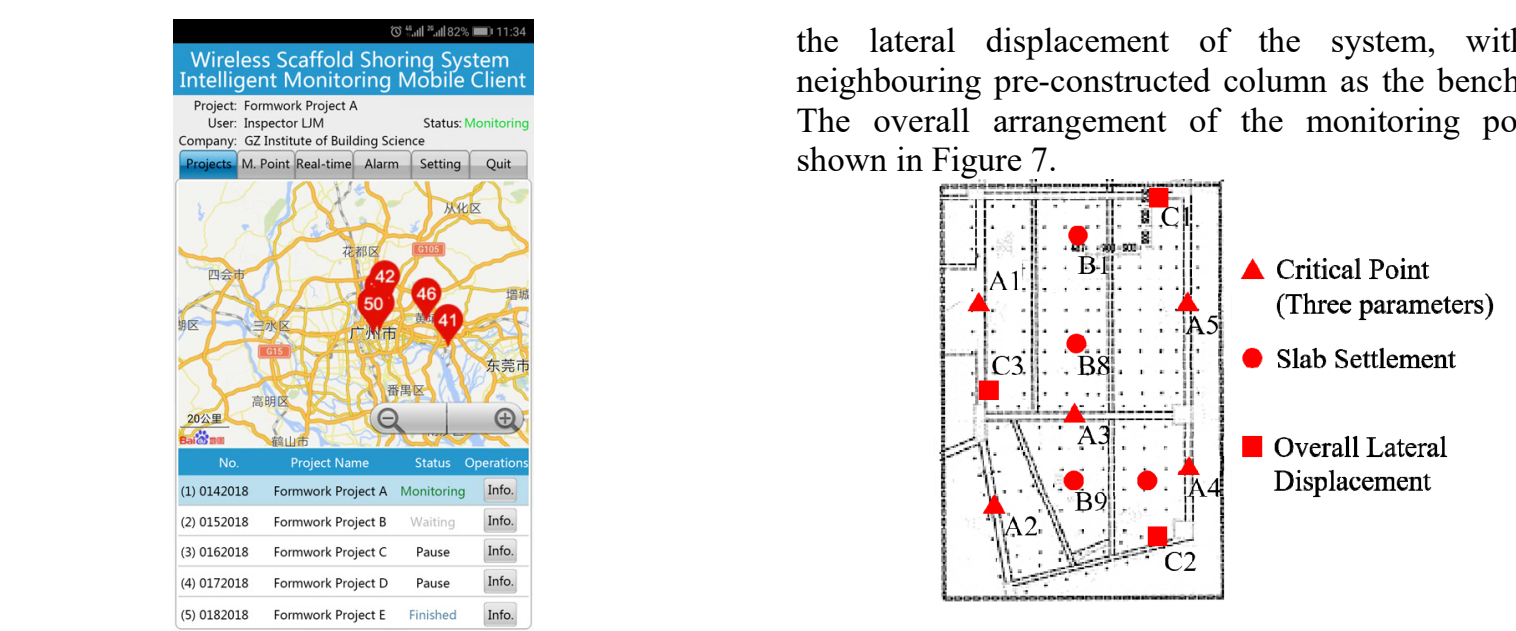

Figure 7. The arrangement of monitoring points

\subsection{The result of monitoring}

The monitoring started at 7:39 am, five minutes later, the concrete placement began, and since then, the collected monitoring data from all sensors started to grow gradually.

By 11:01 am, an unusual situation occurred. The slab settlement at monitoring point A1 increased rapidly and exceeded the alert value $(10 \mathrm{~mm})$ at 11:04, triggering the wireless alarm (Figure 8). The concrete placement was called off immediately and the worker were evacuated at once.

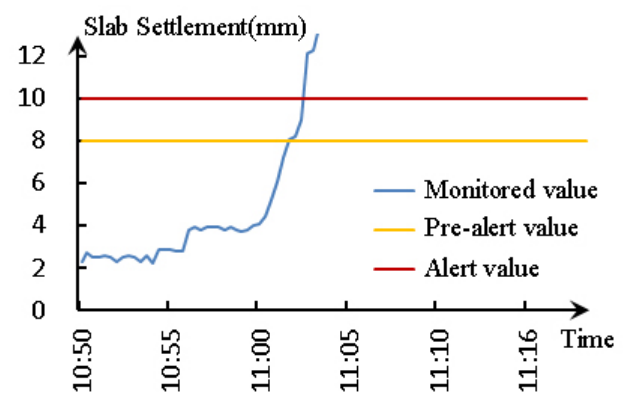

Figure 8. The monitoring data of slab settlement at point A1

After the readings of all sensors became stable, the investigation had found that a slab near point A1 was broken, and the concrete burst out from the gap, as shown in Figure 10. The effectiveness of the Iot-based monitoring system has been proved by this incidence.

\subsection{The layout of monitoring points}

The critical spots for the scaffold shoring system monitoring are where the load is relatively large, in the case of this project, are the middle of beams. Five critical spots have been chosen to be critical monitoring points in which three parameters (slab settlement, axial force and tilt of column) are monitored. To increase the effectiveness of the monitoring, four more points are arranged, where only the slab settlement is monitored. Moreover, at the rim of the scaffold shoring system, three wireless displacement sensors are deployed to monitor 


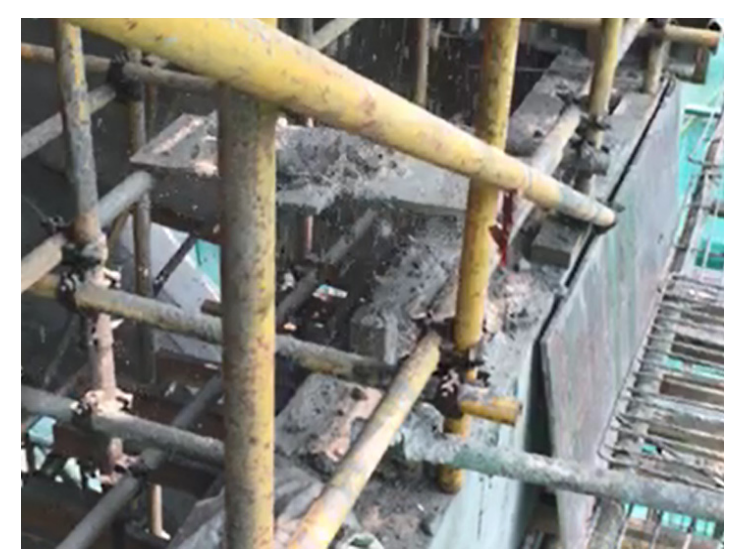

Figure 9. Concrete bursting out

\section{CONCLUSIONS}

(1) The instability of the scaffold and the failure of materials are the two main causes of the collapse of scaffold shoring system during concrete formwork. The instability of the scaffold can be affected by material, designing and assembling factors.

(2) The IoT technology can be applied to the monitoring of scaffold shoring system. The IoT-based monitoring system can get rid of the blind area of optical monitoring and realize real-time monitoring, which significantly enhances the effectiveness.

(3) In the practical application, the breaking of a slab was detected by the monitoring system, proving the validity and reliability of the IoT- bases monitoring system.

\section{ACKNOWLEDGEMENT}

This study was supported by the Guangdong Provincial Science and Technology Foundation (Grant No. 2016A040403067) and Foundation of Guangzhou Science Technology and Innovation Commission (Grant No. 201704020148).

\section{REFERENCES}

1. Q.L. An, J.L. Liu, CQ. Arch. 11, 45 (2012)

2. S.S. Fang, W.Y. Wang, Y. Ying, Const. Tech. 14, 83 (2015)

3. S.Q. Liao, Gd. Bldg. Mat. 27, 34 (2011)

4. S.Y. Lu, L.J. Liang, G.J. He, Bltn. Surv. Mpg. 7, 85 (2015)

5. S. Moon, B. Choi, B. Yang. J. Comput. Civ. Eng. 26, 271 (2012)

6. Y.L. Huang, W.F Chen, H.J. Chen, T. Yen, Y.G. Kao, C.Q. Lin. Comput. Struct. 78, 681 (2000)

7. X.X. Yuan. PH.D Thesis. ZheJ. Un. (2006)

8. C.M. Hu. Spatial stucture failure mechanism and saftey control for high and large tube and coupler falsework (Science Press, Beijing, 2014) 